\title{
MOLECULAR DYNAMICS SIMULATIONS OF CLUSTER IMPACTS
} ON METALLIC TARGETS

MARK H. SHAPIRO* AND T.A. TOMBRELLO**

*Physics Department, California State University, Fullerton, CA $92634-9480$

* Mail Code 200-36, California Institute of Technology, Pasadena, CA 91125 [Supported in part by NSF grant DMR86-15641]

\section{ABSTRACT}

A modified version of the multiple-interaction code SPUT2 has been used to simulate impacts of 63-atom $\mathrm{Cu}$ clusters on six-layer $C u$ targets. Simulations were carried out with cutoff times of 100 and 500 fs for an incident cluster energy of $63 \mathrm{keV}$ (1 kev/atom). Significant enhancements were observed in the maximum potential and kinetic energies achieved in the early phase of the collision cascade. Some hard collisions yielded atoms with potential energies as high as $925 \mathrm{eV}$ (in the CM frame). This is almost twice the energy allowed in an isolated two-body collision. The number of hard collisions per time-step vs potential energy is well-fitted with a decaying exponential, allowing extrapolation to higher energies. These results together with similar results for Al clusters impacting Au targets suggest that non-linear collisional effects cannot explain the high $D-D$ fusion rates seen in Beuhler, Friedlander, and Friedman's recent experiment [1].

\section{INTRODUCTION}

Cluster bombardment of solid surfaces can produce very high transient particle and energy densities in very small regions of a metallic target $[2,3]$. This results from the fact that large clusters (up to a few hundred atoms) with moderate energies ( I $\mathrm{kev} / \mathrm{atom})$ lose most of their energy through elastic nuclear collisions, rather than through electronic processes [3]. Thus, the impact of a cluster on a surface can produce far more localized damage than an individual ion impacting with comparable total energy. Although experimental work with energetic beams of ionized clusters is still in its infancy, a variety of interesting effects have been reported.

The Brookhaven group has used beams of ionized water clusters to create holes and craters with diameters up to $150 \dot{A}$ in carbon targets, and smaller diameter holes and craters in metalic targets $[2,4,5]$. Henkes and Klingelhofer have used 155 $\mathrm{keV} \mathrm{CO}_{2}$ clusters containing about 1000 molecules to carry out micromachining of copper targets [6]. They report that very smooth, highly polished surfaces can be obtained in this manner for a relatively low expenditure of beam energy compared to sputtering with atomic ions. Henkes and Klingelhofer obtained a sputtering yield of approximately $600 \mathrm{Cu}$ atoms per incident cluster, for an energy cost of about $260 \mathrm{eV}$ per atom removed. This must be contrasted with the Brookhaven group's claim of sputtering yields approaching $10^{5}$ for $300 \mathrm{keV}\left(\mathrm{H}_{2} \mathrm{O}\right)_{150^{-\mathrm{H}^{+}}}$clusters impacting thin gold targets $[4,5]$. 
Recently, the Brookhaven group has used $\left(\mathrm{D}_{2} \mathrm{O}\right)_{\mathrm{n}} \mathrm{H}^{+}$clusters with $25 \leq n \leq 1300$ and energies up to $325 \mathrm{keV}$ to bombard $\mathrm{TiD}_{2}$ targets in an attempt to initiate D-D fusion [1]. They claim to have observed $D-D$ fusion at extraordinarily high rates in these experiments $\left(\sim 10^{-11}\right.$ per incident cluster). Since such a high fusion rate would require an unrealistically high fusion cross section for an uncompressed system, they have suggested that the incoming clusters create shock waves that result in significant compression of the cluster and target material [1]. In a separate investigation reported elsewhere [7], we have shown using MD simulations that inertial confinement of cluster impacts on metalilc targets is not a likely candidate to explain the Brookhaven group's results. Recently Echenique, Manson and Ritchie have suggested that multiple collision processes during cluster impacts might produce a sufficent number of high energy particles to explain the high rate of $D-D$ fusion seen by Beuhler et al. [8]. They assumed that the high-energy tail of the velocity distribution in the collision cascade could be represented by a decaying exponential. In their model the fusion rate is proportional to

$$
\int_{0}^{\infty} d E e^{-E / E_{0}} e^{-A / \sqrt{E}}
$$

where they assume $E_{0}=500 \mathrm{eV}$, and $A=31.28 \mathrm{keV}^{1 / 2}$ [1]. However, Echenique et al. are careful to note the sensitivity of the fusion rate to the value of $E_{0}$.

The molecular-dynamics simulations reported in this paper were carried out in an attempt to learn more about the nonlinear properties of the early stages of the collision cascades induced by energetic cluster impacts on metallic targets. The energy properties of the early collision cascade were of particular interest because they might account for the high fusion rate seen in [1]. The Cu-Cu system was chosen for this initial investigation because well established two-body potentials are available for this system [9], along with extensive experimental sputtering data for a wide range of incident atomic and molecular ions [10] in the energy range of interest. Although we have not studied the detailed nuclear physics of cluster impact fusion, our results on the purely collisional aspects of cluster impacts limit the possibility that nonlinear energy effects were responsible for the high fusion rates seen in [1].

\section{SIMULATION MODEL}

The simulations reported in this paper were carried out with a modified version of the molecular-dynamics sputtering code SPUT2 [11]. In this code interactions between atoms are represented by a linear superposition of two-body potentials. A repulsive Moliére potential joined smoothly to an attractive Morse well by a cubic spline was used to describe the interactions between copper atoms. The functional form of the potential and the specific parameters for $\mathrm{Cu}$ are given in [12]. In addition to the usual information about sputtered atoms, 
the modified program computed the total potential energy of each atom in the cluster-target system at each time step. Whenever the total potential energy for an atom exceeded that which could be achieved in an isolated two-body collision with the target atom at rest ( $500 \mathrm{eV}$ for $\mathrm{Cu}-\mathrm{Cu}$ collisions), this information was recorded in a file.

Calculations were carried out for 63-atom Cu clusters impacting normally on Cu targets with $63 \mathrm{keV}$ total energy, i.e. 1 $\mathrm{keV}$ per atom. The targets were six layer, fcc single crystals containing 1875 atoms. They were oriented with their $(1,0,0)$ faces perpendicular to the incoming clusters. For computational simplicity the clusters were chosen to be copper crystallite cubes with their $(1,0,0)$ faces perpendicular to the direction of incidence. The primary impact zone for the clusters was chosen to be a square region at the center of the target, which retained the basic symmetry of the target. This square region was divided into 100 smaller squares which were scanned uniformly. Within each small square the exact impact coordinates were chosen randomly. Three hundred impacts were simulated with a cutoff time of $100 \mathrm{fs}$, and one hundred impacts were simulated with a cutoff time of $500 \mathrm{fs}$.

\section{RESULTS}

\section{Sputtering Yields}

For times up to 100 fs the collision cascade appeared to be well contained within the 1875 atom target. The yield at this point was $\sim 91$ sputtered atoms per cluster impact. By 500 fs there was a substantial loss of containment, and the calculated yield of $\sim 377$ atoms per cluster impact probably was about 608 of the true yield. One particularly interesting result was the low number of sputtered atoms from the vicinity of the primary impact zone. Figure 1 shows sputtering yields as a function of initial position for atoms located along the midlines of the target for both the 100 fs and 500 fs cut-off time simulations.

Apparently, collisions with successive layers of $\mathrm{Cu}$ atoms in the incoming cluster effectively move target atoms in the primary impact region deeper into the target. While these atoms become part of the early, hot collision cascade they do not often sputter.

\section{Sputtered atom energy distributions}

Figure 2 shows the calculated yields of sputtered Cu atoms as a function of energy for both the $100 \mathrm{fs}$ and 500 fs cut-off time simulations. The energy distribution of $\mathrm{Cu}$ target atoms sputtered within the first 100 fs of impact is exceptionally hard. The peak of this distributions occurs at about $7.5 \mathrm{ev}$, which is one indication of the highly nonlinear nature of the early stage of the collision cascade. This distribution exhibits an exponential decay with energy in contrast to the $1 / E^{2}$ distribution usually encountered in sputtering initiated by light, single ions.

By 500 fs enough low energy atoms have been sputtered to 


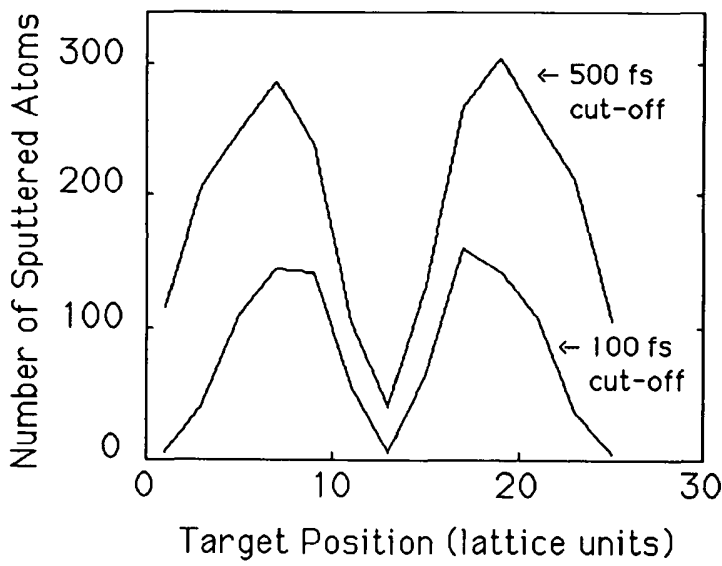

Figure 1. Sputtering yield vs initial location of sputtered atoms for atoms located along the target midlines. Target positions are given in lattice units ( $1 \mathrm{lu}=1.81$ A for $\mathrm{Cu}$ ). Both curves have been normalized to 100 incident cluster impacts.

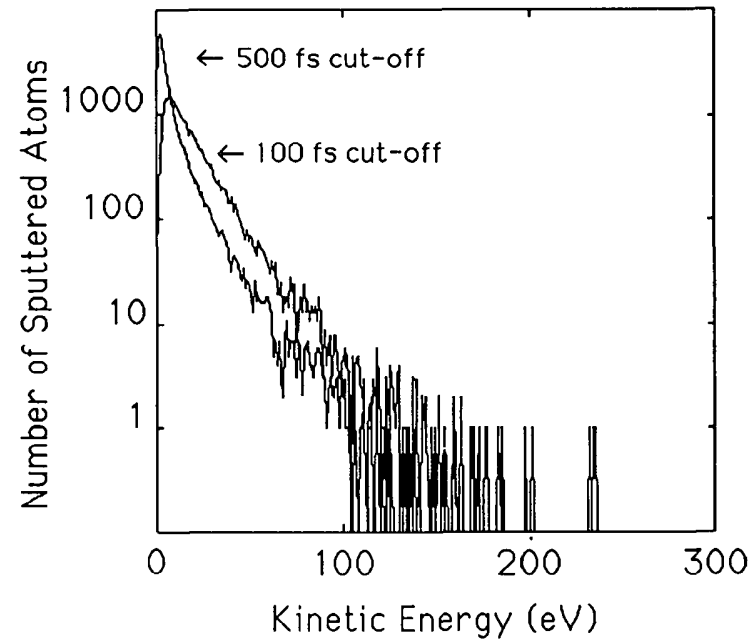

Figure 2. Energy distributions of target atoms sputtered by 100 fs and $500 \mathrm{fs}$. The $100 \mathrm{fs}$ spectrum was calculated from trajectories for 300 incident clusters. The 500 fs spectrum is based on 100 incident clusters.

lower the peak in the energy distribution to about $2.5 \mathrm{eV}$. This is close to the value observed in sputtering induced by $\mathrm{Ar}^{+}$ions [13]; however, the high energy portion of the spectrum remains 
significantly harder than $1 / E^{2}$. Very few sputtered atoms were observed with energies higher than $200 \mathrm{eV}$.

\section{Distribution of potential energies within the collision cascade}

Figure 3 shows the behavior of the potential energy for individual atoms in the simulated collision cascades. The number of time steps for which a given potential energy is reached have been plotted vs the energy value. Because several time steps may be required to complete a collision, this plot overestimates the number of collisions for which a given potential energy is achieved. However, for high energy events the overestimate is small. In this simulation a few collisions yielded potential energies as high as $925 \mathrm{ev}$ - nearly twice that achievable in an isolated two-body collision. The data in Figure 3 are well fitted with a decaying exponential function. The number of events per incident cluster is approximately $N(E)=(1.37 \mathrm{x}$ $\left.10^{4}\right) \exp (-17.04 \mathrm{E})$, where $\mathrm{E}$ is in $\mathrm{keV}$. Thus, only about 1 impact

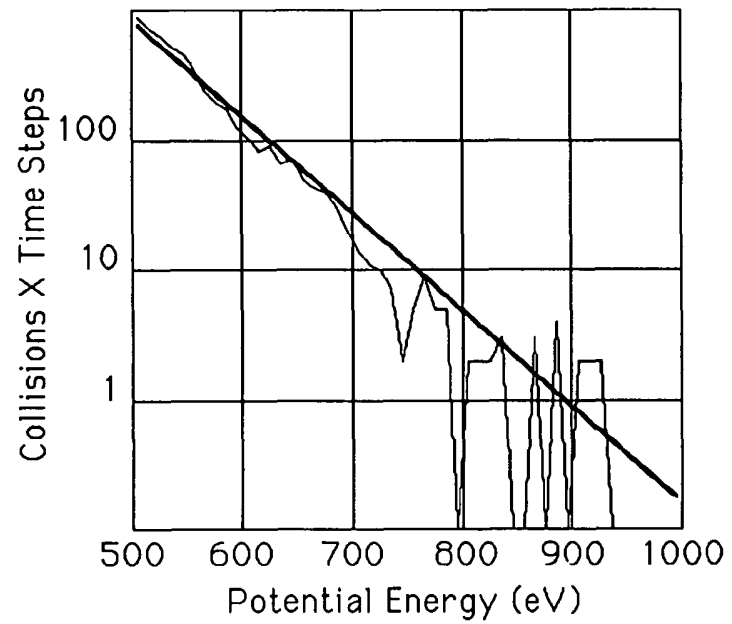

Figure 3. Number of time steps at which the given potential energy per atom was reached for the $\mathrm{Cu}_{63} \mathrm{Cu}$ system. The solid line represents a least squares fit of a decaying exponential to the simulation result (parameters given in the text).

in $10^{24}$ would lead to a potential energy as high as $4 \mathrm{keV}$ for this system.

\section{DISCUSSION}

Our simulation results for the sputtering yields are much closer to the experimental results of Henkes and Klingelhofer [6] than to those of the Brookhaven group $[4,5]$. It is possible that 
in the latter work significant amounts of material were removed in large aggregates from the carbon substrate rather than as individually sputtered atoms and small clusters. This could have occurred by thermal evaporation of individual gold "islands" from the relatively nonconducting carbon substrate.

Our simulation results for the $\mathrm{Cu}_{63^{-}} \mathrm{Cu}$ system show that nonlinear collision processes between moving atoms occasionally increase the energy of an individual atom to as much as twice the incident energy per atom; however, the probability for such events decreases rapidly with energy. Together with similar results found in the $\mathrm{Al}_{63} \mathrm{Au}$ system [7], this would suggest that the high D-D fusion rate found in [1] was not caused by nonlinear collisional effects.

\section{REFERENCES}

1. R.J. Beuhler, G. Friedlander, and L. Friedman, Phys. Rev. Lett. 63, 1292 (1989).

2. R. Beuhler and L. Friedman, Chem. Rev. 86, 521 (1986).

3. P. Sigmund, J. de Physique C2, 175 (1989).

4. M.W. Matthew, R.J. Beuhler, M. Ledbetter, and L. Friedman, J. Phys. Chem. 90, $251(1986)$.

5. M.W. Matthew, R.J. Beuhler, M. Ledbetter, and L. Friedman, Nucl. Inst. and Methods Bl4, 448 (1986).

6. P.R.W. Henkes and R. Klingelhofer, J. de Physique C2, 159 (1989).

7. M.H. Shapiro and T.A. Tombrello, Caltech preprint BB-94, (1990), submitted to Phys. Rev. Lett.

8. P.M. Echenique, J.R. Manson, and R.H. Ritchie, Phys. Rev. Lett. 64, 1413 (1990).

9. D.E. Harrison, Jr., Crit. Rev. in Solid State and Mat. Sci. 14, S1 (1988).

10. R. Berisch, ed., Sputtering by Particle Bombardment, Vol. I, (Springer-Verlag, Basel, 1981).

11. M.H. Shapiro, T.A. Tombrello, and D.E. Harrison, Jr., Nucl. Inst. and Meth. B30, 152 (1988).

12. M.H. Shapiro, D.Y. Lo, P.K. Haff, and T.A. Tombrello, Nucl. Inst. and Meth. B13, 348 (1986).

13. R.A. Brizzolara, C.B. Cooper, and T.K. Olson, Nucl. Inst. and Meth. B35, 36 (1988). 